\title{
Comportamento fenológico de três espécies de Jatropha (Euphorbiaceae) da Caatinga, semi-árido do Brasil ${ }^{1}$
}

\author{
EDINALDO LUZ DAS NEVES ${ }^{2,3}$, LIGIA SILVEIRA FUNCH² e BLANDINA FELIPE VIANA ${ }^{3,4}$
}

(recebido: 07 de agosto de 2008; aceito: 25 de fevereiro de 2010)

\begin{abstract}
Phenological behavior of three species of Jatropha (Euphorbiaceae) in the semi-arid Caatinga region of Brazil). The vegetative and reproductive phenological behavior of Jatropha mollissima (Pohl) Baill., Jatropha mutabilis (Pohl) Baill. and Jatropha ribifolia (Pohl) Baill. were characterized and compared in an area of hyperxerophilous shrub-arboreal caatinga in northeastern Brazil from July 2005 to June 2007. The correlation between the phenophases and the abiotic variables (temperature, relative air humidity, precipitation and photoperiod), the capacity for water storage and the patterns of spatial distribution of these species were also investigated. All species showed high capacities for water storage, continuous and irregular phenological behaviors with flowers, fruits, leaf fall, and leaf budding absence during the coldest and wettest months. Only flowering in J. ribifolia demonstrated significant correlation with precipitation. All species presented aggregated distribution of their populations, which was favored by the ballistic autochory. These results indicated that the high capacity of these species for storing water facilitates the occurrence of phenological events even in the absence of rainfall.
\end{abstract}

Key words - flowering, fruiting, Jatropha, phenology

RESUMO - (Comportamento fenológico de três espécies de Jatropha (Euphorbiaceae) da Caatinga, semi-árido do Brasil). O comportamento fenológico vegetativo e reprodutivo de Jatropha mollissima (Pohl) Baill., Jatropha mutabilis (Pohl) Baill. e Jatropha ribifolia (Pohl) Baill. foi comparado em uma área de caatinga hiperxerófila arbustiva-arbórea do nordeste brasileiro, no período de julho de 2005 a junho de 2007. Adicionalmente, investigou-se a correlação entre as fenofases e as variáveis abióticas (temperatura, umidade relativa, precipitação e fotoperíodo), a capacidade de armazenamento de água e o padrão de distribuição espacial. Todas as espécies apresentaram alta capacidade de armazenamento de água e comportamento fenológico contínuo e irregular com ausência de flores, frutos, queda foliar e brotamento nos meses mais frios e úmidos do ano. Apenas a floração de $J$. ribifolia apresentou correlação significativa com a precipitação. As três espécies apresentaram populações com distribuição agregada, favorecida principalmente devido ao processo de dispersão de sementes por autocoria. Os resultados obtidos indicam que a alta capacidade de armazenamento de água pelas espécies garante a ocorrência dos eventos fenológicos mesmo na ausência de chuvas.

Palavras-chave - fenologia, floração, frutificação, Jatropha

\section{Introdução}

Os estudos sobre fenologia abordam os diferentes eventos biológicos repetitivos que ocorrem durante o ciclo de vida das plantas (e.g. florescimento, frutificação, brotamento, queda foliar e germinação) e as causas de sua ocorrência em relação a fatores bióticos, abióticos, endógenos e restrições filogenéticas (Lieth 1974, Wright \& Calderon 1995, Williams-Linera \& Meave 2002).

1. Parte da tese de doutorado do primeiro autor, Programa de Pósgraduação em Botânica da Universidade Estadual de Feira de Santana (UEFS), Feira de Santana, BA, Brasil.

2. Universidade Estadual de Feira de Santana (UEFS), Departamento de Ciências Biológicas, Rodovia BR116 km 03, Campus Universitário, 44031-460 Feira de Santana, BA, Brasil.

3. Universidade Federal da Bahia (UFBA), Instituto de Biologia, Departamento de Zoologia, Grupo de Ecologia de Comunidades de Visitantes Florais. Rua Barão de Geremoabo, s/n, Campus Universitário de Ondina, 40170-110 Salvador, BA, Brasil.

4._Autor para correspondência: blande.viana@gmail.com
Os eventos fenológicos influenciam a relação planta-planta devido a interações tais como competição por recursos ou por polinizadores. Entretanto, o estudo do comportamento fenológico das plantas torna-se mais relevante devido ao fato dos ciclos reprodutivo e vegetativo afetarem não apenas suas populações, mas também os animais que dependem de suas flores, frutos e demais recursos (Frankie et al. 1974, Newstrom et al. 1994, Leigh 1999).

Estudos indicam que a sobreposição na floração entre espécies simpátricas conduz à partilha e/ou competição por polinizadores (Levin \& Anderson 1970, Sakai 2000, Fonseca et al. 2008). Entre as espécies vegetais taxonomicamente relacionadas, a partilha de polinizadores pode resultar em hibridização, redução do sucesso reprodutivo e perda de pólen (Campbell \& Motten 1985). A competição, por sua vez, pode interferir no número de polinizadores e de visitas às flores $\mathrm{e}$ levar os indivíduos vegetais ao isolamento reprodutivo (Murcia 1995). 
Os ciclos fenológicos de plantas tropicais são pouco estudados (Newstrom et al. 1994) e, além disso, são complexos, apresentando padrões irregulares de difícil reconhecimento, tanto em larga quanto em pequena escala geográfica, principalmente em estudos de curto prazo (Morellato et al. 2000, Bencke \& Morellato 2002).

A ocorrência de eventos fenológicos em ambientes semi-áridos parece ser fortemente influenciada não só pela precipitação (Machado et al. 1997, Bulhão \& Figueiredo 2002), mas também pela disponibilidade de água para a planta, que pode estar relacionada à sua capacidade de armazenar água no caule ou nas raízes em períodos de seca, ou à presença de raízes profundas (Borchert 1994, Borchert \& Rivera 2001, Griz \& Machado 2001).

Um dos fatores bióticos mais fortemente relacionados aos eventos fenológicos reprodutivos é a dispersão. A dispersão corresponde ao deslocamento dos diásporos, através de agentes bióticos ou abióticos, para locais distantes da planta-mãe, seguros, onde a predação e a competição são mais baixos e propícios à germinação (van der Pijl 1982, Howe \& Miriti 2004). Esse processo demográfico é limitante no ciclo de vida das plantas por representar a ponte que une a polinização ao recrutamento, que leva ao estabelecimento de novas plantas adultas (Harper 1977) e influencia o padrão de distribuição espacial das populações (Fonseca 2004).

A Caatinga, bioma característico da região Nordeste do Brasil e padrão de cobertura vegetal mais extenso do estado da Bahia, caracteriza-se por apresentar precipitações escassas e irregulares (Ab'Saber 1974, Andrade-Lima 1981). A despeito da importância biológica da Caatinga, são raros os estudos que abordam eventos fenológicos desse bioma (e.g. Oliveira et al. 1988, Barbosa et al. 1989, Pereira et al. 1989, Machado et al. 1997, Griz \& Machado 2001, Lima 2007).

A família Euphorbiaceae está representada por 317 gêneros e cerca de 8000 espécies distribuídas principalmente em regiões tropicais, nos mais variados tipos de vegetação e habitats (Webster 1994a, b). Na Caatinga, foram registradas 73 espécies (Giulietti et al. 2006), das quais 17 endêmicas (Sampaio et al. 2002), sendo considerada a terceira família com maior riqueza em espécies, superada apenas por Fabaceae e Convolvulaceae.

O gênero Jatropha L. possui cerca de 150 espécies, principalmente nos continentes africano e americano (Webster 1994b). Jatropha mollissima (Pohl) Baill., Jatropha mutabilis (Pohl) Baill. e Jatropha ribifolia (Pohl) Baill. são arbustos ocorrentes da Caatinga. Possuem inflorescência terminal cimosa, monóica, flores pentâmeras, pistiladas e estaminadas do tipo prato e produtoras de néctar e frutos do tipo esquizocárpicos, secos, com três cocas globosas de deiscência explosiva e, conseqüentemente, dispersão primária por autocoria. São raros os estudos sobre a fenologia das espécies de Jatropha. Na Caatinga, destaca-se o trabalho desenvolvido por Santos et al. (2005) que aborda os dados de um ano de amostragem sobre aspectos da biologia floral, sistema reprodutivo e fenologia reprodutiva das espécies $J$. mollissima e $J$. mutabilis nos municípios pernambucanos de Buíque e Arcoverde.

Diante da relevância dos estudos de fenologia para o entendimento das interações planta-animal e do sucesso reprodutivo das espécies vegetais e a escassez de informações acerca das espécies vegetais do Bioma Caatinga, o presente estudo teve por objetivos principais caracterizar e comparar os comportamentos fenológicos vegetativos e reprodutivos das espécies Jatropha mollissima, J. mutabilis e J. ribifolia em uma área de caatinga hiperxerófila arbustiva-arbórea do nordeste brasileiro, no período de julho de 2005 a julho de 2007. Adicionalmente, investigou-se a correlação entre os eventos fenológicos e as variáveis ambientais, a capacidade de armazenamento de água e o padrão de distribuição espacial dessas espécies.

\section{Material e métodos}

Área de estudo - As coletas de dados foram mensais e realizadas no período de julho de 2005 a junho de $2007 \mathrm{em}$ uma área de caatinga hiperxerófila arbustiva-arbórea, na Estação Biológica de Canudos (9॰56’34” S, 3859’17’ W), pertencenteà Fundação Biodiversitas, localizada na Ecorregião do Raso da Catarina (Velloso et al. 2002), área prioritária para a conservação da flora e fauna de invertebrados da Caatinga, de acordo com o relatório do Ministério do Meio Ambiente (2002). A Estação encontra-se a $400 \mathrm{~m}$ de altitude, possui 1.447 ha e foi criada em 1989 com o objetivo de proteger in situ a arara-azul-de-lear Anodorhynchus leari (Bonaparte 1857) (Aves, Psittacidae). Na área de estudo, as chuvas são irregulares e a temperatura média mantém-se alta, exceto no período de maio a agosto, caracterizando o período mais frio e úmido do ano (figura 1). Os dados de temperatura, umidade relativa e precipitação foram obtidos no INMET 4o Distrito e os de fotoperíodo foram calculados a partir de Lammi (2005).

Coleta de dados fenológicos - Foram selecionados 20 indivíduos adultos e saudáveis de cada espécie, distantes pelo menos 200 metros entre si. Os indivíduos foram etiquetados e receberam numeração definitiva. Para cada indivíduo, estimou-se o percentual de flores estaminadas, flores pistiladas, frutos, brotamento e queda foliar, seguindo 


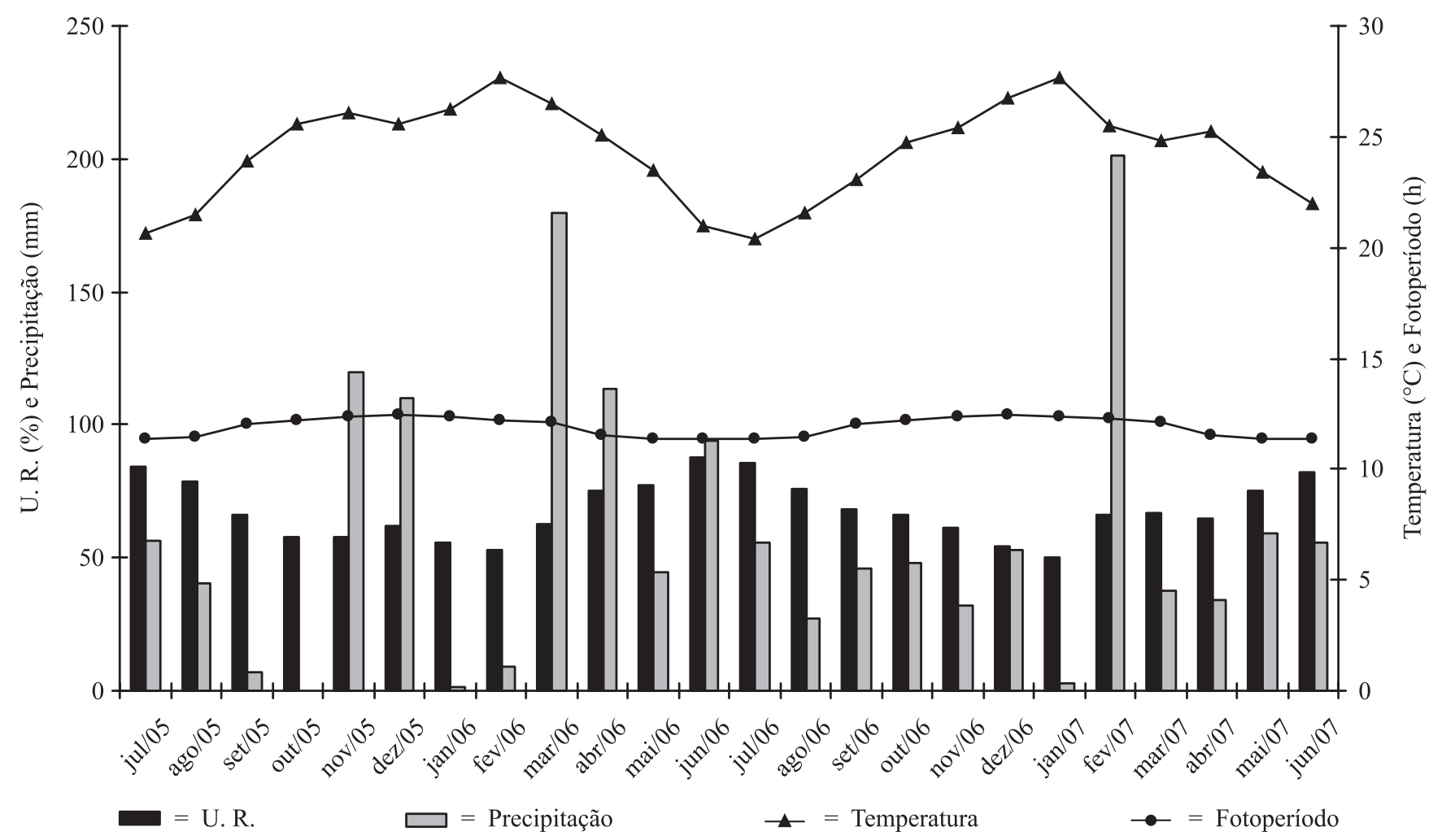

Figura 1. Dados de Umidade Relativa - UR (\%), Precipitação $(\mathrm{mm})$, Temperatura $\left({ }^{\circ} \mathrm{C}\right)$ e Fotoperíodo (h) do município de Monte Santo, inserida na Ecorregião do Raso da Catarina. Dados fornecidos pelo INMET - IV Distrito para o período de julho de 2005 a junho de 2007.

Figure 1. Relative Humidity - UR (\%), Precipitation (mm), Temperature $\left({ }^{\circ} \mathrm{C}\right)$, and Photoperiod $(\mathrm{h})$ of the municipality of Monte Santo, within the Raso da Catarina Eco-region, Bahia State, Brazil. Data provided by INMET - District IV, from July 2005 to June 2007.

a escala semiquantitativa de Fournier (1974). Os dados fenológicos foram coletados no período das $6 \mathrm{~h} 00$ às 09h30min, período anterior ao início da abscisão das flores estaminadas. Os padrões de floração e frutificação foram determinados segundo Newstrom et al. (1994). Para avaliar o grau de deciduidade das três espécies, seguiu-se Williams et al. (1997). O material botânico coletado (E.L. Neves 1, 2, 3) encontra-se depositado no Herbário da Universidade Estadual de Feira de Santana (HUEFS), sob os números 134859,134860 e 134861.

Para estimar a porcentagem de intensidade da fenofase em cada indivíduo utilizou-se a análise de intensidade de Fournier (1974). Para cada mês, fez-se a soma dos valores de intensidade obtidos para todos os indivíduos de cada espécie e dividiu-se pelo valor máximo possível (número de indivíduos multiplicado por 4). O valor obtido, que corresponde a uma proporção, foi então multiplicado por 100, para transformá-lo em valor percentual.

Mediu-se a presença ou ausência da fenofase no indivíduo utilizando-se o índice de atividade proposto por Bencke e Morellato (2002). Esse índice indica a percentagem de indivíduos na população que está manifestando determinado evento fenológico e a sincronia entre os indivíduos da população.

O grau de sobreposição das fenofases entre os pares de espécie foi medido utilizando-se a fórmula proposta por Krebs (1989):

$$
\mathrm{P} j k=\left[\sum^{\mathrm{n}} \text { (mínimo Pij, Pik) }\right] 100
$$

Onde:

$\mathrm{P} j k=$ porcentagem de sobreposição da fenofase entre as espécies $j$ e $k$;

$\mathrm{P} i j, \mathrm{P} i k=$ proporção do recurso $i$ em cada mês em relação ao recurso total ofertado pelas espécies $j$ e $k$ no período estudado;

$\mathrm{n}=$ total de datas amostradas.

Analisou-se a correlação de cada fenofase com os dados de precipitação, temperatura, umidade relativa e fotoperíodo, aplicando-se o índice de correlação de Spearman (Zar 1996), com nível de significância de 0,05 . Os cálculos foram feitos utilizando-se o programa estatístico SPSS for Windows versão 8. Para controlar o acúmulo de erros oriundos dos intervalos de 
confiança de cada variável, utilizou-se a correção de Bonferroni, dividindo-se o nível de significância por $4(0,0125)$.

Densidade básica da madeira - Para a análise do grau de densidade da madeira, seguiu-se o modelo proposto por Borchert et al. (2002). Adensidade básica da madeira (DBM) é a relação entre a massa seca (MS) e o volume saturado da madeira (VS) (Trugilho et al. 1990). Utilizando-se outros cinco indivíduos adultos de cada espécie, coletou-se quatro discos amostrais (casca + alburno + cerne) de cada indivíduo, sendo um de cada ponto cardinal da planta, com 5 a $8 \mathrm{~cm}$ de comprimento e diâmetros variáveis, totalizando 20 discos amostrais. Após coleta, ainda em campo, cada disco foi mergulhado em água durante três dias para saturação, depois teve sua massa saturada $\left(\mathrm{M}_{\text {sat }}\right)$ medida. Após essa etapa, cada peça foi mantida em repouso por um período de 5-10 minutos para escorrer o excesso de água e, em seguida, foi mergulhada em provetas graduadas de $250 \mathrm{~mL}$ para determinação do deslocamento do líquido provocado pela imersão da peça (Trugilho et al. 1990). Logo depois, cada peça foi seca em estufa até alcançar a massa seca constante (MS), considerando-se duas medidas seguidas com massas iguais - a primeira realizada $24 \mathrm{~h}$ após o início da secagem e as seguintes, com intervalos de seis horas.

A densidade básica da madeira (DBM) e a quantidade de água saturada $\left(\mathrm{QA}_{\text {sat. }} \%\right)$ foram calculadas de acordo com as fórmulas abaixo.

$$
\operatorname{DBM}\left(\mathrm{g} \mathrm{cm}^{-3}\right)=\operatorname{MS}(\mathrm{g}) / \mathrm{VS}\left(\mathrm{cm}^{3}\right)
$$

Onde:

$\mathrm{VS}=\left(\pi \mathrm{r}^{2}\right) \mathrm{h}$

$\mathrm{h}=$ altura do deslocamento do líquido

$\mathrm{MS}=$ massa seca da madeira

$\mathrm{r}=$ raio da proveta graduada

$\mathrm{VS}=$ volume saturado da madeira

$$
\mathrm{QA}_{\text {sat }} \%=100\left(\mathrm{M}_{\text {sat. }}(\mathrm{g})-\mathrm{MS}(\mathrm{g})\right) / \mathrm{MS}(\mathrm{g})
$$

Onde:

$\mathrm{M}_{\text {sat. }}=$ massa saturada

A quantidade de água saturada $\left(\mathrm{QA}_{\text {sat. }} \%\right)$ representa a capacidade de armazenamento de água da madeira, de acordo com Borchert (1994).

Padrão de distribuição espacial - Para a coleta de dados necessários à análise do padrão de distribuição espacial (Ludwig \& Reynolds 1988) das três espécies de Jatropha, demarcou-se uma transecção de $800 \mathrm{~m}$, de onde foram definidas sete bases amostrais, cada uma delas originando quatro quadrantes. Em cada quadrante foram feitas as seguintes medidas: $(\mathrm{x})$ representando o primeiro indivíduo encontrado a partir da base e (y) representando o indivíduo vizinho mais próximo.
Para calcular o índice de distribuição espacial (C) das três espécies de Jatropha, utilizou-se o programa Basic Tsquare. bas. De acordo com esse índice, para o padrão aleatório, o valor de $\mathrm{C}$ esperado é próximo a 0,5 ; para o padrão uniforme, significantemente menor do que 0,5 e para o padrão agregado, significantemente maior que 0,5 . A significância estatística de z sobre $C$ é obtida através de uma tabela de probabilidade para distribuição normal, considerando-se $\mathrm{z}=1,96$ para $P=0,05$ (Ludwig \& Reynolds 1988).

\section{Resultados}

De acordo com as figuras 2,3 e 4, pode-se inferir que as três espécies apresentaram comportamento fenológico contínuo e variável quanto à freqüência, duração e regularidade dos episódios nos anos estudados.

As figuras 2, 3 e 4 mostram que, para as três espécies, os percentuais de intensidade e de atividade foram maiores para as flores estaminadas durante a maior parte do tempo. Houve ausência dos dois tipos de flores (estaminadas e pistiladas) nos meses mais frios e úmidos e de flores pistiladas em momentos isolados do ciclo fenológico. A frutificação apresentou comportamento fenológico contínuo no primeiro episódio e anual no segundo, sendo que houve uma tendência à ocorrência de frutos nos períodos de picos pluviométricos.

Para J. mollissima e J. mutabilis, o brotamento tendeu a ocorrer depois do período de queda foliar, mas ocorreram eventos de sobreposição dessas fenofases. Para J. ribifolia, ao contrário das demais espécies, houve maior ocorrência de brotamento do que de queda foliar. De agosto de 2006 a junho de 2007, quando se observa maior freqüência de precipitação, mesmo com baixos índices na maioria dos meses, a fenofase de brotamento foi observada continuamente.

As três espécies apresentaram comportamento foliar decíduo, já que todos os indivíduos ficaram sem folhas por, pelo menos, um mês. Os indivíduos apresentaram deciduidade total entre maio e julho, meses com temperaturas mais baixas e dias mais curtos. Jatropha mollissima e $J$. mutabilis apresentaram um segundo período de deciduidade total entre os meses de setembro e outubro. Em J. mutabilis observou-se a formação de botões e início de abertura das flores antes do amadurecimento das primeiras folhas.

Não houve correlação significativa entre a precipitação e as fenofases de $J$. mollissima (tabela 1) e J. mutabilis (tabela 2). Para J. ribifolia, a precipitação influenciou fortemente a produção de flores estaminadas e pistiladas (tabela 3). Para J. mollissima, a produção de flores estaminadas foi fortemente influenciada pela 

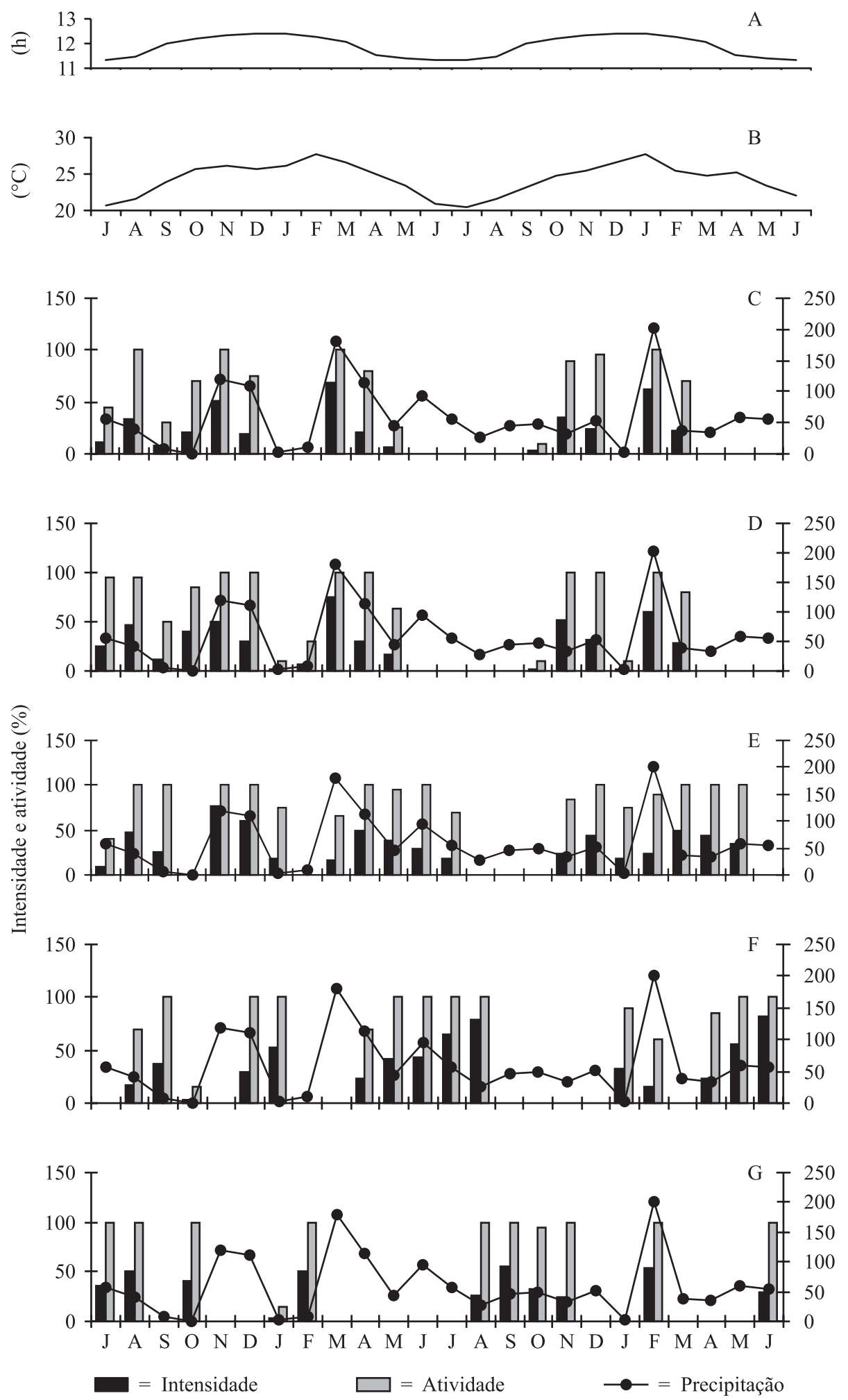

Figura 2. Comparação entre os percentuais de intensidade e de atividade de Jatropha mollissima e os dados meteorológicos da Ecorregião do Raso da Catarina no período de julho de 2005 a junho de 2007. (A. Comprimento do dia. B. Temperatura. C. Flores pistiladas. D. Flores estaminadas. E. Frutificação. F. Queda foliar. G. Brotamento).

Figure 2. Comparisons between the percentages of intensity and activity of Jatropha mollissima and meteorological data from the Raso da Catarina Eco-region from July 2005 to June 2007. (A. Day length. B. Temperature. C. Pistillate flowers. D. Staminate flowers. E. Fruiting. F. Leaf fall. G. Leaf budding). 

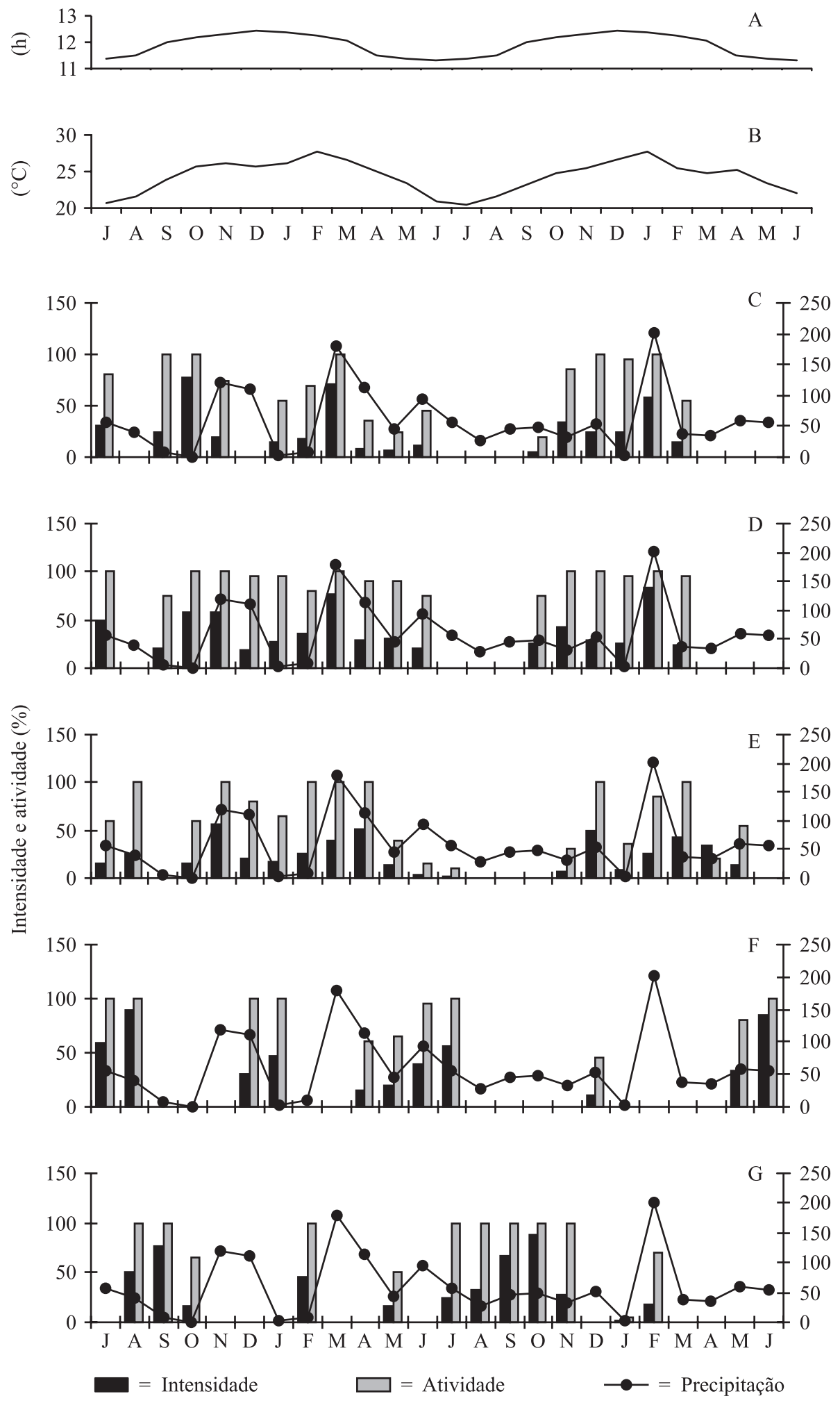

Figura 3. Comparação entre os percentuais de intensidade e de atividade de Jatropha mutabilis e os dados meteorológicos da Ecorregião do Raso da Catarina no período de julho de 2005 a junho de 2007. (A. Comprimento do dia. B. Temperatura. C. Flores pistiladas. D. Flores estaminadas. E. Frutificação. F. Queda foliar. G. Brotamento).

Figure 3. Comparisons between the percentages of intensity and activity of Jatropha mutabilis and meteorological data from the Raso da Catarina Eco-region from July 2005 to June 2007. (A. Day length. B. Temperature. C. Pistillate flowers. D. Staminate flowers. E. Fruiting. F. Leaf fall. G. Leaf budding). 

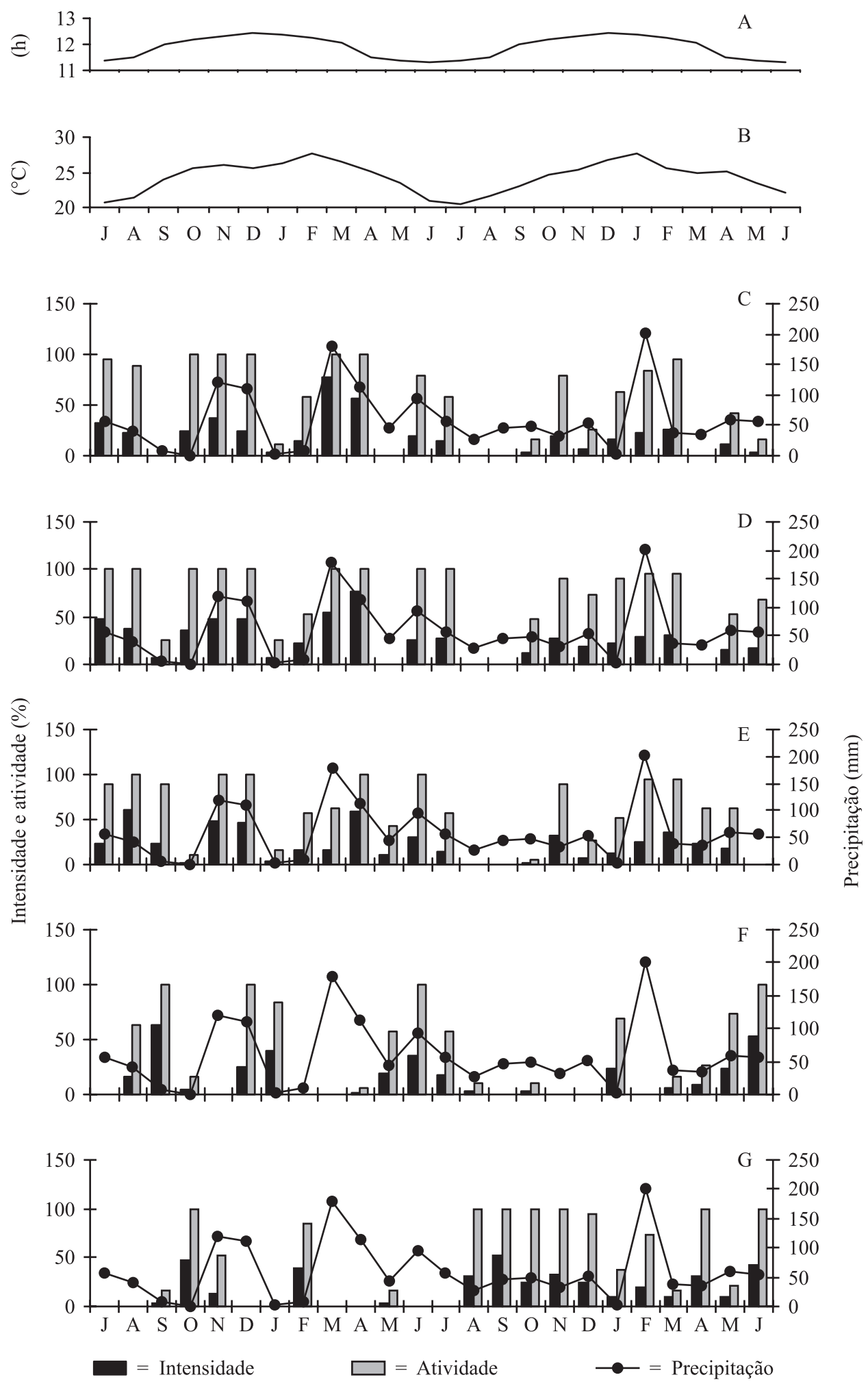

Figura 4. Comparação entre os percentuais de intensidade e de atividade de Jatropha ribifolia e os dados meteorológicos da Ecorregião do Raso da Catarina no período de julho de 2005 a junho de 2007. (A. Comprimento do dia. B. Temperatura. C. Flores pistiladas. D. Flores estaminadas. E. Frutificação. F. Queda foliar. G. Brotamento).

Figure 4. Comparisons between the percentages of intensity and activity of Jatropha ribifolia and meteorological data from the Raso da Catarina Eco-region from July 2005 to June 2007. (A. Day length. B. Temperature. C. Pistillate flowers. D. Staminate flowers. E. Fruiting. F. Leaf fall. G. Leaf budding). 
Tabela 1. Resultados do coeficiente de correlação de Spearman entre as fenofases de Jatropha mollissima e os fatores abióticos.

Table 1. Results of Spearman's correlation coefficient relating the phenophases of Jatropha mollissima and abiotic factors.

\begin{tabular}{lllll}
\hline \multirow{2}{*}{ Fenofase } & \multicolumn{4}{c}{ Fatores abióticos } \\
\cline { 2 - 5 } & Precipitação & Temperatura & Umidade Relativa & Fotoperíodo \\
\hline Flores pistiladas & $\mathrm{rs}=0,366$ & $\mathrm{rs}=0,292$ & $\mathrm{rs}=-0,205$ & $\mathrm{rs}=0,346$ \\
Flores estaminadas & $\mathrm{rs}=0,258$ & $\mathrm{rs}=0,524^{*}$ & $\mathrm{rs}=-0,427$ & $\mathrm{rs}=0,536^{*}$ \\
Frutos & $\mathrm{rs}=0,326$ & $\mathrm{rs}=0,104$ & $\mathrm{rs}=-0,021$ & $\mathrm{rs}=0,130$ \\
Brotamento & $\mathrm{rs}=-0,200$ & $\mathrm{rs}=-0,132$ & $\mathrm{rs}=0,037$ & $\mathrm{rs}=0,024$ \\
Queda foliar & $\mathrm{rs}=-0,239$ & $\mathrm{rs}=-0,314$ & $\mathrm{rs}=0,309$ & $\mathrm{rs}=-0,393$ \\
\hline
\end{tabular}

$* P<0,05=$ Valores significativos segundo o teste de correlação de Spearman. $* P<0,05=$ Significative values according to the Spearman rank correlation test.

Tabela 2. Resultados do coeficiente de correlação de Spearman entre as fenofases de Jatropha mutabilis e os fatores abióticos.

Table 2. Results of Spearman's correlation coefficient relating the phenophases of Jatropha mutabilis and abiotic factors.

\begin{tabular}{lclcc}
\hline \multirow{2}{*}{ Fenofase } & \multicolumn{4}{c}{ Fatores abióticos } \\
\cline { 2 - 5 } & Precipitação & Temperatura & Umidade Relativa & Fotoperíodo \\
\hline Flores pistiladas & $\mathrm{rs}=-0,057$ & $\mathrm{rs}=0,522^{*}$ & $\mathrm{rs}=-0,484$ & $\mathrm{rs}=0,435$ \\
Flores estaminadas & $\mathrm{rs}=0,165$ & $\mathrm{rs}=0,557^{*}$ & $\mathrm{rs}=-0,452$ & $\mathrm{rs}=0,439$ \\
Frutos & $\mathrm{rs}=0,308$ & $\mathrm{rs}=0,531^{*}$ & $\mathrm{rs}=-0,379$ & $\mathrm{rs}=0,363$ \\
Brotamento & $\mathrm{rs}=-0,434$ & $\mathrm{rs}=-0,144$ & $\mathrm{rs}=-0,022$ & $\mathrm{rs}=0,083$ \\
Queda foliar & $\mathrm{rs}=0,243$ & $\mathrm{rs}=-0,516^{*}$ & $\mathrm{rs}=0,573^{*}$ & $\mathrm{rs}=-0,487$ \\
\hline
\end{tabular}

* $P<0,05=$ Valores significativos segundo o teste de correlação de Spearman. $* P<0,05=$ Significative values according to the Spearman rank correlation test.

Tabela 3. Resultados do coeficiente de correlação de Spearman entre as fenofases de Jatropha ribifolia e os fatores abióticos.

Table 3. Results of Spearman's correlation coefficient relating the phenophases of Jatropha ribifolia and abiotic factors.

\begin{tabular}{llccc}
\hline \multirow{2}{*}{ Fenofase } & \multicolumn{4}{c}{ Fatores abióticos } \\
\cline { 2 - 5 } & Precipitação & Temperatura & Umidade Relativa & Fotoperíodo \\
\hline Flores pistiladas & $\mathrm{rs}=0,517^{*}$ & $\mathrm{rs}=0,211$ & $\mathrm{rs}=-0,054$ & $\mathrm{rs}=0,138$ \\
Flores estaminadas & $\mathrm{rs}=0,503^{*}$ & $\mathrm{rs}=0,146$ & $\mathrm{rs}=-0,013$ & $\mathrm{rs}=0,115$ \\
Frutos & $\mathrm{rs}=0,386$ & $\mathrm{rs}=0,023$ & $\mathrm{rs}=-0,058$ & $\mathrm{rs}=0,058$ \\
Brotamento & $\mathrm{rs}=-0,375$ & $\mathrm{rs}=0,204$ & $\mathrm{rs}=-0,327$ & $\mathrm{rs}=0,183$ \\
Queda foliar & $\mathrm{rs}=-0,390$ & $\mathrm{rs}=-0,357$ & $\mathrm{rs}=-0,289$ & $\mathrm{rs}=-0,344$ \\
\hline
\end{tabular}

* $P<0,05=$ Valores significativos segundo o teste de correlação de Spearman. $* P<0,05=$ Significative values according to the Spearman rank correlation test.

temperatura e pelo fotoperíodo. Para J. mutabilis, a temperatura influenciou fortemente as fenofases, exceto o brotamento; a umidade relativa, a queda foliar; e o fotoperíodo, a produção de flores pistiladas.
Considerando-se o total de indivíduos apresentando uma determinada fenofase, os pares de espécies apresentaram altos índices de sobreposição para todos os eventos estudados (tabela 4). Os percentuais continuaram 
Tabela 4. Percentuais de sobreposição entre os pares de espécies de Jatropha, considerando-se o total de indivíduos apresentando determinada fenofase.

Table 4. Percentage overlap between pairs of species of Jatropha, considering the total of individuals of each phenophase.

\begin{tabular}{|c|c|c|c|c|c|c|c|c|}
\hline \multirow[t]{2}{*}{ Espécies } & \multicolumn{4}{|c|}{ J. mollissima } & \multicolumn{4}{|c|}{ J. mutabilis } \\
\hline & Flor & Fruto & Brotamento & Queda foliar & Flor & Fruto & Brotamento & Queda foliar \\
\hline J. ribifolia & 72 & 85 & 67 & 77 & 77 & 77 & 59 & 69 \\
\hline J. mollissima & - & - & - & - & 75 & 73 & 73 & 64 \\
\hline
\end{tabular}

Tabela 5. Percentuais de sobreposição entre os pares de espécies de Jatropha, considerando-se o grau de intensidade de cada fenofase.

Table 5. Percentage overlap between pairs of species of Jatropha, considering the degree of intensity of each phenophase.

\begin{tabular}{|c|c|c|c|c|c|c|c|c|}
\hline \multirow[t]{2}{*}{ Espécies } & \multicolumn{4}{|c|}{ J. mollissima } & \multicolumn{4}{|c|}{ J. mutabilis } \\
\hline & Flor & Fruto & Brotamento & Queda foliar & Flor & Fruto & Brotamento & Queda foliar \\
\hline J. ribifolia & 66 & 76 & 65 & 71 & 68 & 69 & 52 & 60 \\
\hline J. mollissima & - & - & - & - & 75 & 73 & 62 & 63 \\
\hline
\end{tabular}

altos quando foram analisados os graus de sobreposição entre os pares em relação ao grau de intensidade de cada fenofase (tabela 5). Analisou-se, também, o grau de sobreposição de floração e de frutificação para as três espécies simultaneamente e verificaram-se altos percentuais de sobreposição. Considerando-se o total de indivíduos apresentando a fenofase, obteve-se $64 \%$ de sobreposição na floração e $66 \%$ de sobreposição na frutificação. Levando-se em consideração o grau de intensidade de cada fenofase, obteve-se $55 \%$ de sobreposição na floração e $57 \%$ na frutificação.

As três espécies apresentaram valores próximos de densidade básica da madeira. Quanto à capacidade de armazenamento de água, registraram-se os maiores valores para $J$. mutabilis $\left(\mathrm{DBM}=0,342 \pm 0,077 \mathrm{~g} \mathrm{~cm}^{-3}\right.$ $\mathrm{QA}_{\text {sat. }}=339,87 \pm 72,77 \%$ ), seguida por $J$. ribifolia $\left(\mathrm{DBM}=0,332 \pm 0,046 \mathrm{~g} \mathrm{~cm}^{-3} ; \mathrm{QA}_{\text {sat. }}=291,47 \pm 43,43 \%\right)$ e J. mollissima $\left(0,321 \pm 0,056 \mathrm{~g} \mathrm{~cm}^{-3} ; \mathrm{QA}_{\text {sat. }}=287,61 \pm\right.$ $31,37 \%)$.

As três espécies investigadas apresentaram o padrão de distribuição espacial agregado. Os valores obtidos para o Índice de Distribuição Espacial (Ludwig \& Reynolds 1988) foi de $\mathrm{C}=0,88, \mathrm{z}=6,88$ para J. mollissima; $\mathrm{C}=0,83, \mathrm{z}=6,01$ para $J$. mutabilis e $\mathrm{C}=0,87, \mathrm{z}=6,86$ para $J$. ribifolia. Nas populações das três espécies, observaram-se sementes germinando próximas às plantas-mães e as plântulas desenvolvendo-se até a idade adulta, quando iniciaram a produção de flores.

\section{Discussão}

A variação geográfica nos padrões fenológicos dentro de uma mesma espécie é mais comum nos trópicos do que nas zonas temperadas já que os padrões fenológicos nos trópicos são mais diversos (Newstrom et al. 1994). Quando se compara os resultados obtidos no presente estudo com aqueles apresentados por Santos et al. (2005) para as espécies J. mollissima e J. mutabilis, percebe-se que nem todos os eventos apresentaram comportamentos semelhantes. Essa diferença deve estar associada a variações existentes entre as duas localidades quanto à intensidade e distribuição pluviométrica e ao comprimento do dia, fatores que influenciam o comportamento fenológico das plantas.

Santos et al. (2005) encontraram o padrão fenológico contínuo para as espécies $J$. mollissima e $J$. mutabilis, com a floração estendendo-se durante todo o ano e o pico de atividade nos meses de outubro e novembro e os menores percentuais de atividade nos meses de agosto e setembro. Entretanto, no presente estudo, as populações apresentaram ausência de floração durante quatro meses consecutivos (junho a setembro), nos meses mais frios e curtos, em J. mollissima e três meses (julho a setembro) em $J$. mutabilis e de frutificação, em três meses consecutivos nas duas espécies (agosto a outubro). Jatropha ribifolia apresentou ausência dessas fenofases em apenas dois meses consecutivos (agosto e setembro). 
O comportamento de floração obtido para as três espécies no presente estudo apresenta maior semelhança àquele observado para J. mollissima por Machado et al. (1997) na Serra Talhada, PE, e Lima (2007) em Betânia e Floresta, PE, onde os indivíduos não permaneceram floridos nem produziram frutos durante todo o ano.

Santos et al. (2005) registraram a maior produção de flores pistiladas durante o período mais frio e de dias mais curtos, enquanto no presente estudo não houve produção de flores nesse período. Além disso, o fato dos indivíduos das três espécies estudadas no presente estudo terem permanecido desfolhadas por mais de um mês, caracteriza-as como caducifólia, enquanto aqueles autores observaram o comportamento perenifólio para $J$. mollissima e J. mutabilis. O comportamento caducifólio para J. mollissima também foi observado por Lima (2007).

Os resultados obtidos no presente estudo indicam que as três espécies estudadas possuem madeira de baixa densidade e, conseqüentemente, alta capacidade de armazenamento de água nos tecidos. Essa alta capacidade de armazenamento de água pode ter favorecido a ocorrência dos eventos fenológicos vegetativos e reprodutivos, mesmo na ausência de precipitação, corroborando a hipótese de que plantas que armazenam muita água nos seus tecidos podem brotar e florescer durante a estação seca (Borchert 1994, Chapotin et al. 2006). Resultado semelhante foi obtido por Lima (2007), em que todos os táxons que brotaram, floresceram e frutificaram na estação seca possuíam madeira de baixa densidade.

A correlação existente entre a precipitação e a produção de flores em $J$. ribifolia pode indicar que essa espécie, devido ao seu menor porte em relação às duas outras estudadas (Neves 2008), é mais dependente das chuvas para o desenvolvimento de eventos fenológicos reprodutivos. Esse resultado está de acordo com a hipótese de Seghieri et al. (1995) de que plantas de pequeno porte são mais afetadas pela variação climática devido à menor capacidade de manter armazenados água e nutrientes de reserva. Resultados semelhantes foram obtidos por Mantovani e Martins (1988), Batalha e Mantovani (2000) e Munhoz e Felfili (2005) para espécies herbáceas e subarbustivas do Cerrado.

Alguns autores sugerem que a sobreposição de floração sempre induz à diminuição na produção de frutos devido à competição por polinizadores e à perda de pólen nas flores de outras espécies (e.g. Campbell 1985, Murcia \& Feinsinger 1996). Entretanto, esse parece não ser o caso das espécies estudadas no presente trabalho, já que são polinizados principalmente por Apis mellifera, muito abundante na área (Neves 2008). Além disso, Jatropha ribifolia, na ausência de polinizadores, pode ser auto-polinizada (Neves 2008).

Outra conseqüência da sobreposição de floração para espécies simpátricas é a possibilidade de existência de fluxo gênico (Tiffin et al. 2001, Coyne \& Orr 2004) e conseqüente formação de híbridos naturais, fato relativamente comum entre as angiospermas (Ellstrand et al. 1996). A existência de sobreposição de floração indica que as espécies aqui estudadas não apresentam mecanismos de isolamento temporal que atuem como barreira pré-polinização, o que pode favorecer o aumento do fluxo gênico entre as espécies e conseqüente produção de híbridos, caso não haja barreiras pós-zigóticas.

A dispersão de sementes integra o ciclo reprodutivo das plantas e pode ter importantes conseqüências para a demografia e estrutura genética das populações (Jordano \& Godoy 2002). A dispersão por autocoria parece ser uma estratégia vantajosa para o sucesso reprodutivo das três espécies estudadas, assim como para muitas outras espécies ocorrentes na caatinga. Barbosa et al. (2006) na revisão sobre a fenologia de espécies lenhosas da caatinga, verificaram que $47 \%$ das espécies tiveram os diásporos dispersados por autocoria.

O padrão de distribuição agregado das populações das três espécies parece ser favorecido pela dispersão primária por autocoria balística. Esse padrão de distribuição proporciona a concentração de recursos florais, facilitando a localização destes pelos potenciais polinizadores, entretanto, dificulta a ocupação de novos territórios pelos diásporos e intensifica a ação de predadores de sementes.

Agradecimentos - Os autores agradecem à Fundação Biodiversitas, pela autorização concedida para o desenvolvimento dos trabalhos de campo na Estação Biológica de Canudos; à Fundação de Amparo à Pesquisa do Estado da Bahia - Fapesb (Termo de outorga TO 261/03 Etapa II), pelo apoio financeiro a parte das atividades de campo; ao $\mathrm{CNPq}$, pela bolsa de produtividade a Blandina F. Viana e aos membros do Labea, pelo auxílio nas coletas de campo.

\section{Referências bibliográficas}

AB'SABER,A.N. 1974. O domínio morfoclimático semi-árido das caatingas brasileiras. Geomorfologia 43:1-39.

ANDRADE-LIMA, D. 1981. The caatinga dominium. Revista Brasileira de Botânica 4:149-153.

BARBOSA, D.C.A., ALVES, J.L.H., PRAZERES, S.M. \& PAIVA, A.M.A. 1989. Dados fenológicos de 10 espécies arbóreas de uma área de caatinga (Alagoinha-PE). Acta Botanica Brasilica 3:109-117. 
BARBOSA, D.C.A., BARBOSA, M.C.A. \& LIMA, L.C.M. 2006. Fenologia de espécies lenhosas da caatinga. In Ecologia e conservação da Caatinga (I.R. Leal, M. Tabarelli \& J.M.C. Silva, orgs.). Editora Universitária da UFPE, Recife. p.657-693.

BATALHA, M.A. \& MANTOVANI, W. 2000. Reproductive phenological patterns of cerrado plant species at the Péde-Gigante Reserve (Santa Rita do Passa Quatro, SP, Brazil): a comparasion between herbaceous and wood floras. Revista Brasileira de Biologia 60:129-145.

BENCKE, S.C. \& MORELLATO, L.P.C. 2002. Comparação de dois métodos de avaliação da fenologia de plantas, sua interpretação e representação. Revista Brasileira de Botânica 25:269-275.

BORCHERT, R. 1994. Soil and steam water storage determine phenology and distribution of tropical dry forest trees. Ecology 75:1437-1449.

BORCHERT, R. \& RIVERA, G. 2001. Photoperiodic control of seasonal development and dormancy in tropical stem succulent trees. Tree Physiology 21:213-221.

BORCHERT, R., RIVERA, G. \& HAGNAUER, W. 2002. Modification of vegetative phenology in a tropical semi-deciduous forest by abnormal drought and rain. Biotropica 34:27-39.

BULHÃO, C.F. \& FIGUEIREDO, P.S. 2002. Fenologia de leguminosas arbóreas em uma área de cerrado marginal no nordeste do Maranhão. Revista Brasileira de Botânica 25:361-369.

CAMPBELL, D.R. 1985. Pollinator sharing and seed set of Stellaria pubera: competition for pollination. Ecology 66:544-553.

CAMPBELL, D.R. \& MOTTEN, A.F. 1985. The mechanism of competition for pollination between two forest herbs. Ecology 66:554-563.

CHAPOTIN, S.M., RAZANAMEHARIZAKA, J.H. \& HOLBROOK, M. 2006. Baobab trees (Adansonia) in Madagascar use stored water to flush new leaves but not to support stomatal opening before the rain season. New Phytologist 169:549-559.

COYNE, J.A. \& ORR, H.A. 2004. Speciation. Sinauer, Sunderland.

ELLSTRAND, N.C., WHITKUS, R. \& RIESEBERG, L.H. 1996. Distribution of spontaneous hybrids. Proceedings of the National Academy of Sciences of the United States of America 93:5090-5093.

FONSECA, R.B.S. 2004. Fenologia reprodutiva e dispersão de Melocatus glaucescens Buining \& Brederoo e $M$. paucispinus G. Heimen \& R. Paul (Cactaceae) no município de Morro do Chapéu, Chapada Diamantina, Bahia, Brasil. Dissertação de mestrado, Universidade Estadual de Feira de Santana, Feira de Santana.

FONSECA, R.B.S., FUNCH, L.S. \& BORBA, E.L. 2008. Reproductive phenology of Melocactus (Cactaceae) species from Chapada Diamantina, Bahia, Brazil. Revista Brasileira de Botânica 31:237-244.
FOURNIER, L.A. 1974. Um método cuantitativo para la medición de características fenológicas en árboles. Turrialba 24:422-423.

FRANKIE, G.W., BAKER, H.G. \& OPLER, P.A. 1974. Comparative phenological studies of trees in tropical wet and dry forests in the lowlands of Costa Rica. Journal of Ecology 62:881-919.

GIUliETTI, A.M., CONCEIÇÃO, A. \& QUEIROZ, L.P. 2006. Diversidade e caracterização das fanerógamas do semi-árido brasileiro. Associação Plantas do Nordeste, Recife, v.1.

GRIZ, L.M.S. \& MACHADO, I.C.S. 2001. Fruiting phenology and seed dispersal syndromes in caatinga, a tropical dry forest in the northeast of Brazil. Journal of Tropical Ecology 17:303-321.

HAPER, J.L. 1977. Population biology of plants. Academic Press, London.

HOWE, H.F. \& MIRITI, M.N. 2004. When seed dispersal matters. BioScience 54:651-660.

JORDANO, P. \& GODOY, J.A. 2002. Frugivore-generated seed shadows: a landscape view of demographic and genetic effects. In Frugivores and seed dispersal: ecological, evolutionary and conservation (D.J. Levey, W. Silva \& M. Galetti, eds.). CAB, International Wallingford, p.305-321.

KREBS, C.J. 1989. Ecological methodology. Harper and Row Publishers, New York.

LAMMI, J. 2005. Online protoperiod calculator. http://www. nic.fi/ benefon/sun.php3. (acesso em 05/03/2008).

LEIGH, E.G. 1999. Tropical forest ecology: a view from Barro Colorado Island. Oxford University Press, Oxford.

LEVIN, D.A. \& ANDERSON, W.W. 1970. Competition for pollinators between simultaneously flowering species. American Naturalist 104:345-354.

LIETH, H. 1974. Introduction to phenology and the modeling of seasonality. In Phenology and seasonality modeling (H. Lieth, ed.). Ecological Studies, Springer-Verlag, Berlin, v.8, p.3-19.

LIMA, A.L.A. 2007. Padrões fenológicos de espécies lenhosas e cactáceas em uma área do semi-árido do Nordeste brasileiro. Dissertação de mestrado, Universidade Federal Rural de Pernambuco, Recife.

LUDWIG, J.A. \& REYNOLDS, J.F. 1988. Statistical ecology: a primer methods and computing. John Wiley \& Sons, New York.

MACHADO, I.C.S., BARROS, L.M. \& SAMPAIO, V.S.B. 1997. Phenology of Caatinga species at Serra Talhada, PE, northeastern Brasil. Biotropica 29:57-68.

MANTOVANI, W. \& MARTINS, F.R. 1988. Variações fenológicas das espécies do Cerrado da Reserva Biológica de Mogi-Guaçu, estado de São Paulo. Revista Brasileira de Botânica 23:227-237.

MINISTÉRIO DO MEIO AMBIENTE. 2002. Avaliação e ações prioritárias para a conservação da biodiversidade da caatinga. MMA, Brasília. 
MORELLATO, L.P.C., TALORA, D.C., TAKAHASI, A., BENCKE, C.C., ROMERA, E.C. \& ZIPARRO, V.B. 2000. Phenology of Atlantic Rain Forest trees. Biotropica 32:811-823.

MUNHOZ, C.B.R. \& FELFILI, J.M. 2005. Fenologia do estrato herbáceo-subarbustivo de uma comunidade de campo sujo na Fazenda Água Limpa no Distrito Federal, Brasil. Acta Botanica Brasilica 19:979-988.

MURCIA, C. 1995. Forest fragmentation and the pollination of neotropical plants. In Forest patches in tropical landscapes (J. Schellas \& R. Greenberg, eds.). Island Press, London, p.19-36.

MURCIA, C. \& FEINSINGER, P. 1996. Interspecific pollen loss by hummingbirds visiting flower mixtures: effects of floral architecture. Ecology 77:550-560.

NEVES, E.L. 2008. Polinização de espécies nativas da Caatinga e o papel da abelha exótica Apis mellifera L. Tese de doutorado, Universidade Estadual de Feira de Santana, Feira de Santana.

NEWSTROM, L.E., FRANKIE, G.W. \& BAKER, H.G. 1994. A new classification for plant based on flowering patterns in Lowland Tropical Rain Forest trees at La Selva, Costa Rica. Biotropica 26:141-159.

OLIVEIRA, J.G.B., QUESADO, H.L.C., NUNES, E.P. \& VIANA, F.A. 1988. Observações preliminares da fenologia de plantas da caatinga na Estação Ecológica de Aiuba. Coleção Mossorossense n.538, série B. ESAM, Mossoró.

PEREIRA, R.M.A., ARAÚJO-FILHO, J.A., LIMA, R.V., PAULINO, F.D.G., LIMA, A.O.N. \& ARAÚJO, Z.B. 1989. Estudos fenológicos de algumas espécies lenhosas e herbáceas da caatinga. Ciência Agronômica 20:11-20.

SAKAI, S. 2000. Reproductive phenology of gingers in a lowland mixed dipterocarp forest in Borneo. Journal of Tropical Ecology 16:337-354.

SAMPAIO, E.V.S.B, GIULIETTI, A.M., VIRGÍLIO, J. \& GAMARRA-ROJAS, C.F.L. 2002. Vegetação \& flora da Caatinga. Associação Plantas do Nordeste, Recife.
SANTOS, M.J., MACHADO, I.C. \& LOPES, A.V. 2005. Biologia reprodutiva de duas espécies de Jatropha L. (Euphorbiaceae) em Caatinga, Nordeste do Brasil. Revista Brasileira de Botânica 28:361-373.

SEGHIERI, J., FLORET, C. \& PONTANIER, R. 1995. Plant phenology in relation to water availability: herbaceous and woody species in the savannas of northern Cameroon. Journal of Tropical Ecology 11:237-254.

TIFFIN, P.M., OLSON, S. \& MOYLE, L.C. 2001. Asymetrical crossing barriers in angiosperms. Proceedings of the Royal Society of London 268:861-867.

TRUGILHO, P.F., SILVA, D.A., FRAZÃO, F.J.L. \& MATOS, J.L.M. 1990. Comparação de métodos de determinação da densidade básica em madeira. Acta Amazonica 20:307-319.

VAN DER PIJL, L. 1982. Principles of dispersal in higher plants. Springer-Verlag, New York.

VELLOSO, A.L., SAMPAIO, E.V.S.B. \& PAREYN, F.G.C. (EDS.). 2002. Ecorregiões: propostas para o Bioma Caatinga. TNC-Brasil, Associação Plantas do Nordeste, Recife.

WEBSTER, G.L. 1994a. Classification of the Euphorbiaceae. Annals of the Missouri Botanical Garden 81:3-32.

WEBSTER, G.L. 1994b. Synopsis of the genera and suprageneric taxa of Euphorbiaceae. Annals of the Missouri Botanical Garden 81:33-144.

WILLIAMS, R.J., MYERS, B.A., MULLER, W.J., DUFF, G.A. \& EAMUS, D. 1997. Leaf phenology of wood species in a north Australian tropical savanna. Ecology 78:2542-2558.

WILLIAMS-LINERA, G. \& MEAVE, J.A. 2002. Patrones fenológicos de bosque lluvioso neotropical de bajura. In Ecologia de bosques lluviosos Neotropicales (M. Guarijuata \& G. Kattan, eds.). Libro Universitario Regional, Costa Rica, p.407-431.

WRIGHT, S.J. \& CALDERON, O. 1995. Phylogenetic patterns among tropical flowering phenologies. Journal of Ecology 83:937-948.

ZAR, J.H. 1996. Biostatistical analysis. Prentice-Hall, New Jersey. 